\title{
Autoimmune Encephalitis
}

National Cancer Institute

\section{Source}

National Cancer Institute. Autoimmune Encephalitis. NCI Thesaurus. Code C122414.

Inflammation of the brain secondary to an immune response triggered by the body itself. 